\title{
Safety on Teleoperation Demining wheeled robots based on fuzzy logic controller and haptic system
}

\author{
Yekkehfallah Majid \\ School of electronic and information engineering, Xi'an Jiaotong University, Xi'an 710049, China \\ Yuanli Cai*, Guao Yang, Naebi Ahmad, Zolghadr Javad \\ School of electronic and information engineering, Xi'an Jiaotong University, Xi'an 710049, China \\ Majid.fallah@stu.xjtu.edu.cn,ylicai@mail.xjtu.edu.cn, gayang@mail.xjtu.edu.cn,ahmad.naebi@stu.xjtu.edu.cn, \\ javad.zolghadr@gmail.com
}

\begin{abstract}
In the teleoperation robots one of the big challenges is operator's error in urgent situations that can makes big problem for robot and other systems which working beside the robot. This paper proposes an approach of haptic system and fuzzy logic system with implementation on teleoperation demining robot. In order to reduce operator's error through fusion of haptic system and fuzzy logic controller, the haptic system conveys vibration to user's hand and fuzzy controller, controls rate of the vibration in different situation to user hand and controls the speed of the robot.
\end{abstract}

Keywords: Haptic system, fuzzy logic, demining robot, vibrotactile, magnetic field.

\section{Introduction}

Task of demining robots is detects the mine in landmine and marks it with tools that mounted on the robot [1] shown in Fig. 1. Currently robots control by human operators with visual display and operators are limited in their interaction with the robot and because of difficult situation and the low threshold for dealing on demining tasks [2] shown in Fig. 2. Minimizing operator's error while care for high level of accuracy and speed is also difficult, if operator can not clearly hear or see data from the mine detector sensor (MDS) in operator GUI or for some other problems as communication delay between operator and robot so the robot enters to hazardous region shown in Fig. 2. For these reasons this paper uses vibrotactile feedback according to the level of MDS output to inform user when robot is near dangerous touch with environment.

Accordingly, there investigates the use of a force three-dimensional joystick for applying forces and vibrations on user's hand. This joystick connected to operator computer in Fig. 5, the joystick haptic device also used to controls the robot speed and robot navigation [3].

This paper presents a fuzzy logic system (FLS) based force feedback generation method for demining robots in teleoperation manipulators deminer robot. The approach utilizes the MDS output and position of arm to generate desired vibrotactile using FLS.

This paper investigates problems of previous work on demining robot such as safety controlling, communication time delays, communication fail, and operator's error. The structure of this paper is in the following: in section 2 will be described previous work on demine robot, in section 3 this paper explained new approach in detail. In section 4 where we implemented FLS and haptic system in MATLAB fuzzy tools, while in section 5 we concluded result of implementation in section 4.

\section{Background}

\subsection{Deminer robot}

Basically mobile deminer teleoperation robot uses for detecting the mine in landmine with MDS mounted

\footnotetext{
* Corresponding author.
} 
on the robot and user controls this robot by joystick, but user just send command to robot by joystick and monitoring robot is through visual display and sound without any repulsive feedback from MDS in urgent situations shown in Fig. 2.

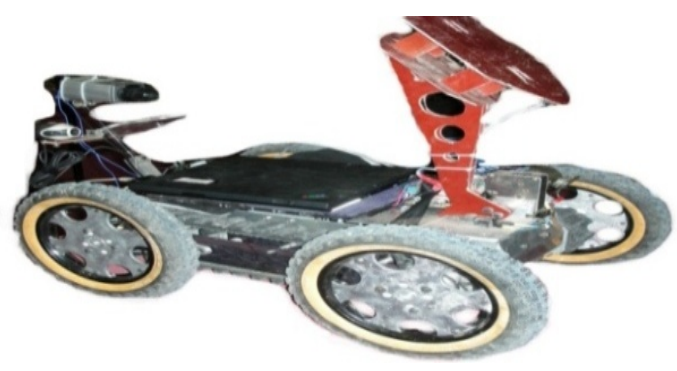

Fig. 1: photograph of the deminer robot.

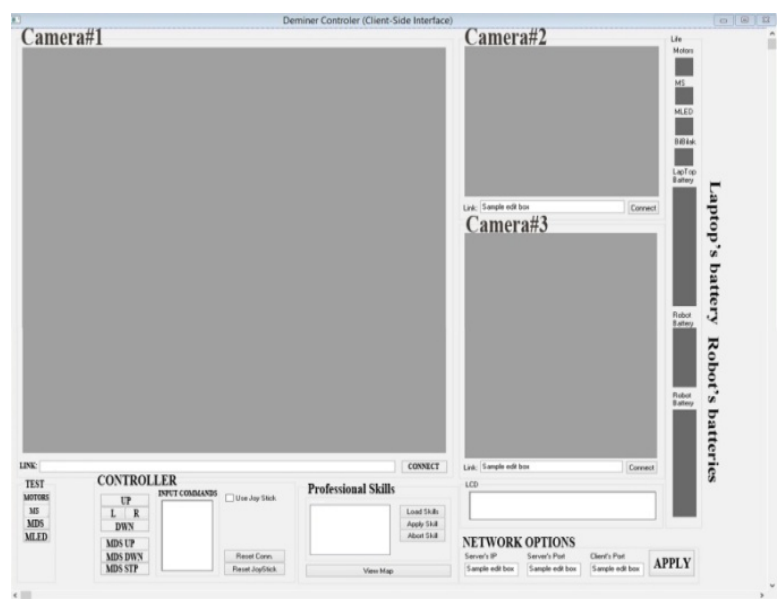

Fig. 2: operator GUI which contained: camera monitoring, robot controller, mine illustrates, network monitoring, robot's batteries status illustrates, laptop's battery status illustrates, motors statues indicators.

This console developed in windows and this is a graphical consol between operator and robot, this console installed in operator computer shown in Fig. 5.

\subsection{Mine Detector sensor (MDS)}

MDS works on the principle of transmitting a magnetic field and analyzing a return signal from the target and environment. These systems may use a single coil as both transmitter and receiver, or they may have two or even three coils working together. In this project, we used two coils first coil is transmitter and second one is receiver signal as shown in Fig. 3.

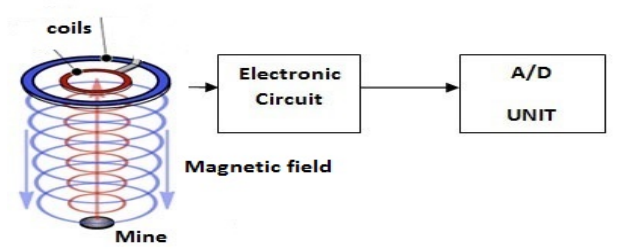

Fig 3: Overall hardware of mine detector sensor

\subsection{Electromagnetic induction}

As mentioned before, in this section there are two parts first one is transmitter and second one is receiver we simulated this two coils with FEMM [4][5] shown in Fig.4. FEMM is one of power full software that uses for designing and analyzing magnetic problems [6][7].

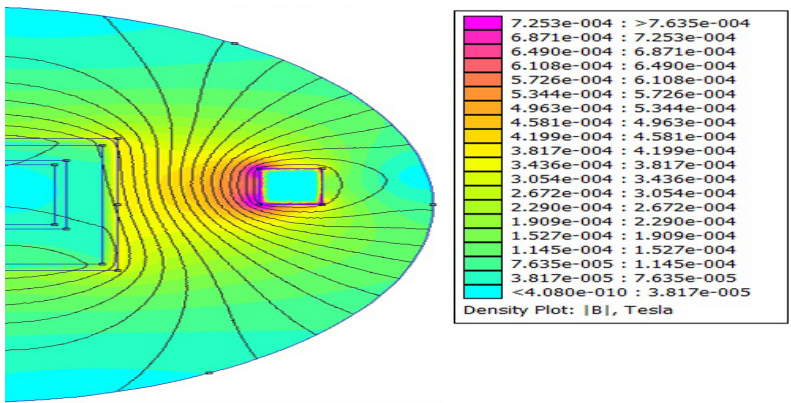

Fig. 4: color flux density plot of two coils with the mine. We added a mine in analyzed region, most of flux passes through the mine because reluctance of the mine is smaller than air therefore, most flux passed through low reluctance and least flux passes through receiver coil and caused least EMF induces in receiver coil.

\section{Haptic system and fuzzy logic controller}

\subsection{New approach}

Our solutions for those problems we mentioned in previous section 2.1 are haptic system and fuzzy logic controller. Haptic system solves feedback problem we suggest two ways for using haptic system in this project the first one is vibrating electric motors in joystick and second suggestion is haptic force three-dimensional joystick actually this joystick is a haptic system contains the vibration electric motors and joystick shown in Fig. 5. Haptic increases human sensitivity from environment and user's hand can feel environment through vibrotactile feedback.

The level of vibrotactile with respect to different situations as MDS sense, robot's speed and robot's arm status is variable, for controlling level of vibrations and velocity of the robot used fuzzy logic controller. 
Operator Side

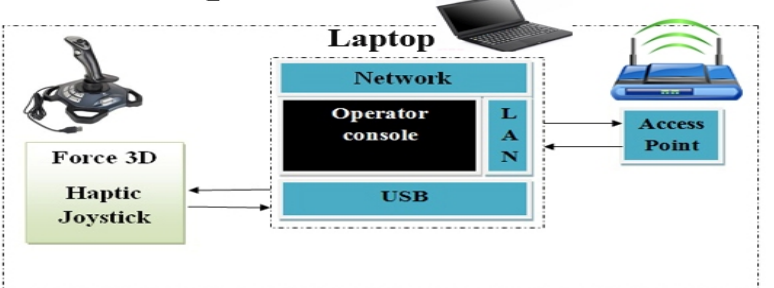

Fig. 5: overall framework of operator side controller. In new approach added force three-dimensional haptic joystick in this part.

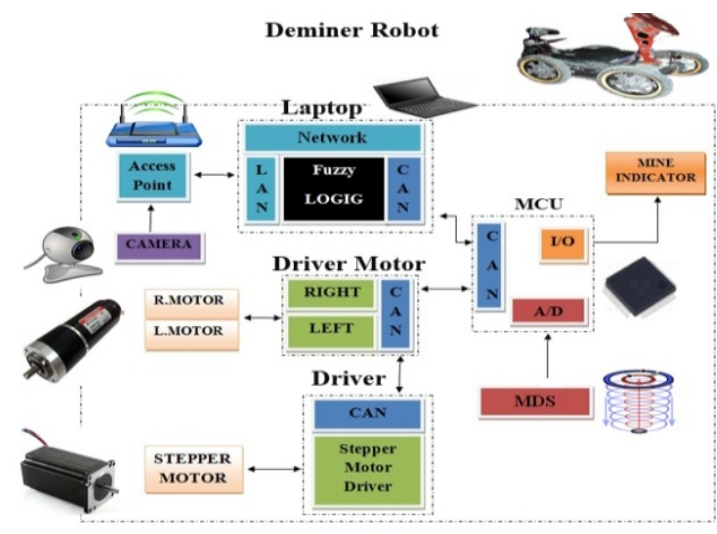

Fig. 6: overall framework of demining robot.

\section{Implementation}

Trap membership function used for MDS inputs and outputs in two FLSs shown in Fig. 8 and Fig. 10 respectively. The rules based in this work are shown in Table 1 and Table 2 the commonly used Madman's min-max implication function was utilized. The control surface generated by the rule base and the given fuzzy sets is depicted in Fig. 9 and Fig. 11 respectively. Detail of fuzzy logic system could find in literatures [8] [9].

\subsection{Fuzzy logic system for dealing with haptic system}

Table1: Rule table of the vibrotactile controller with respect to MDS output and arm status.

\begin{tabular}{|c|c|c|c|c|}
\hline $\begin{array}{c}\text { MDS } \\
\text { OUTPUT }\end{array}$ & $\begin{array}{c}\text { ARM } \\
\text { STATUS }\end{array}$ & DP & OAP & RTP \\
\hline VH & VF & M & VS \\
\hline H & F & M & VS \\
\hline M & M & M & VS \\
\hline L & S & M & VS \\
\hline VL & VS & S & VS \\
\hline
\end{tabular}

Respectively, they are decomposed into five fuzzy partitions for output, such as very low (VL), low (L), medium (M), high $(\mathrm{H})$, very high $(\mathrm{VH})$, and three partition for arm status. As Down position (DP), obstacle avoidance position (OAP), rest time position
(RTP) in last portions related to vibrotactile signal as very slow (VS), slow $(\mathrm{S})$, medium $(\mathrm{M})$, fast $(\mathrm{F})$, very fast (VF) and nothing (NOP).

Since one input divided to five fuzzy sets and another one divided to three fuzzy sets, thus fifteen fuzzy rules for vibrotactile controller must determined. Following, the output signal of MDS directly related to distance between coil and the mine in the ground.

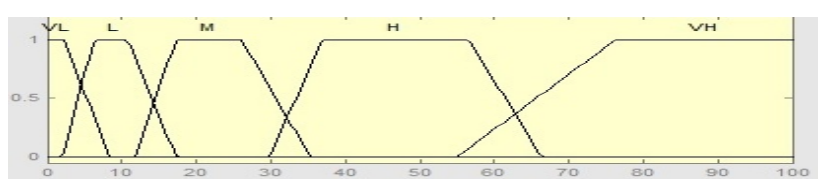

(a)

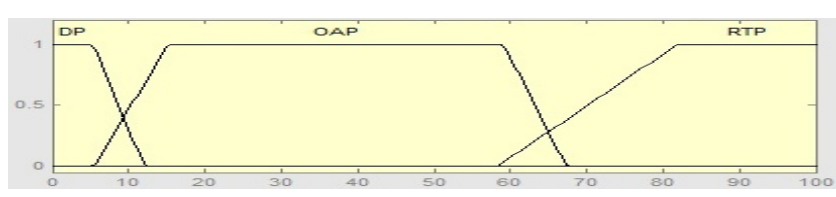

(b)

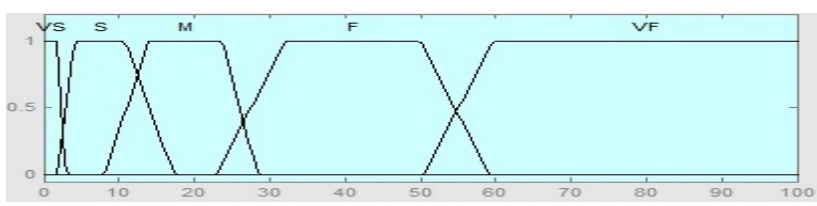

(c)

Fig. 7: (a) membership functions of MDS. (b) Membership functions of arm status. (c) Membership functions of vibrotactile.

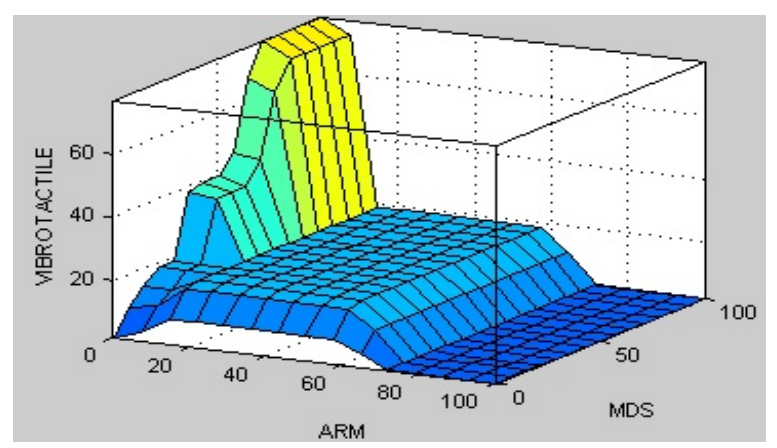

Fig. 8: The output surface of the implemented fuzzy vibrotactile, generator.

Three-dimensional curve that represents mapping from MDS and arm to level of the vibration, because this curve represents a two-input. And one output case as this curve is obvious when the arm is in down position and MDS level is increases therefore, vibrotactile corresponding with MDS level increases. 


\subsection{Fuzzy logic system for velocity controller}

This section is similar to section 4.1 inputs of FLS are same as last one just output is different.

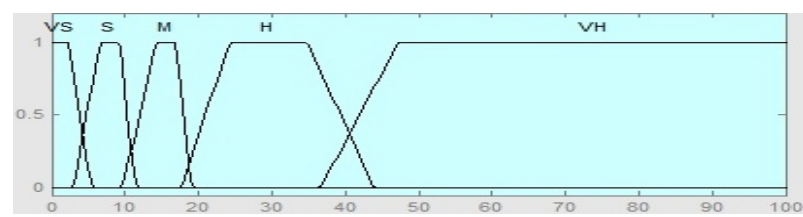

Fig. 9: Membership function of velocity.

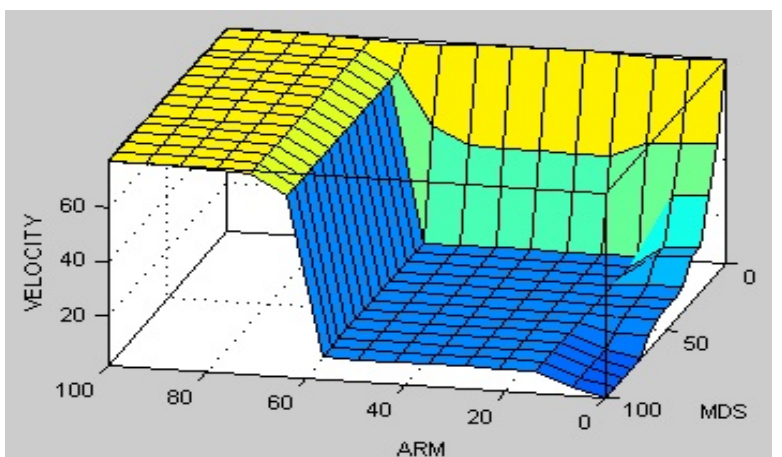

Fig 10: The output surface of the implemented fuzzy velocity controller.

Three-dimensional curve that represents mapping from MDS and arm to robot velocity, because this curve represents, the two-input and one output case. As this curve there is obvious when the arm is in down position and MDS level is increasing in other side velocity with respect to MDS's level reduces.

\section{Conclusion}

This paper presents a dynamic, real-time fuzzy logic based force feedback control for demining robot in remotely operated robot manipulators. The presented method utilizes distance from the mine to the robot, it needs consider two items first one is mine distance to robot and second one is arm's position with respect to these items, and new approach generates force feedback and control speed of the robot. The presented method is implemented on a deminer robot and we also used MATLAB to simulate and illustrates result of vibrotactile data and velocity controller.

The results show that fuzzy logic controller and haptic system, significantly reduced operator's fault and also fuzzy logic prevents dangerous contact between the robot and the mine. When network connection drops, with this method operator drives robot with confidence because this system warns the operator in urgent situations.

\section{Acknowledgment}

This work supported in part by grant from the XI'AN JIOTONG UNIVERSITY and MRL laboratory of QIAU, and the National Natural Science Foundation of Chinese under grants 61573272.

\section{References}

1. Sibert, J., Cooper, J., Covington, C., Stefanovski, A., Thompson, D., and Lindeman, R.W. Vibrotactile feedback for enhanced control of urban search and rescue robots. Proceedings of the IEEE Symposium on Safety, Security and Rescue Robots, Gaithersburg MD, USA, August 22-24, 2006

2. Smith, F. M., D. K. Backman, and Stephen C. Jacobsen Telerobotic manipulator for hazardous environments. Journal of Robotic Systems, pp.251-260, vol.9, no.2, 1992.

3. Pantelios, Michael, et al. Haptics technology in Educational Applications, a Case Study. JDIM, pp.171178, vol. 2, no.4, 2004.

4. Meeker, David. FEMM 4.2 Electrostatics Tutorial1. 2006.

5. Jin, Jian-Ming. The finite element method in electromagnetics. John Wiley \& Sons, 2014.

6. Turner, Robert. Gradient coil design: a review of methods. Magnetic Resonance Imaging, pp.903-920, vol.11, no.7, 1993.

7. Singh, Isha, and Rajul Mishra. Analysis of Magnetic Field of Air Cored Solenoid using FEMM. 2012.

8. Mizumoto, Masaharu, and Hans-Jürgen Zimmermann. Comparison of fuzzy reasoning methods. Fuzzy sets and systems, pp.253-283, vol. 8, no.3, 1982.

9. Iancu, Ion. A Mamdani type fuzzy logic controller. INTECH Open Access Publisher, 2012.

10. Smith F M, Backman D K, Jacobsen S C. Telerobotic manipulator for hazardous environments. Journal of Robotic Systems, pp.251-260, vol.9, no.9, 1992. 